\title{
Single-Shot Single-Gate rf Spin Readout in Silicon
}

\author{
P. Pakkiam, A. V. Timofeev, M. G. House, M. R. Hogg, T. Kobayashi, M. Koch, S. Rogge, and M. Y. Simmons \\ Australian Research Council Centre of Excellence for Quantum Computation and Communication \\ Technology, School of Physics, UNSW Sydney, New South Wales 2052, Australia
}

(Received 10 September 2018; revised manuscript received 16 October 2018; published 26 November 2018)

\begin{abstract}
For solid-state spin qubits, single-gate rf readout can minimize the number of gates required for scale-up since the readout sensor can integrate into the existing gates used to manipulate the qubits. However, stateof-the-art topological error correction codes benefit from the ability to resolve the qubit state within a single shot, that is, without repeated measurements. Here, we demonstrate single-gate, single-shot readout of a singlet-triplet spin state in silicon, with an average readout fidelity of $82.9 \%$ at $3.3 \mathrm{kHz}$ measurement bandwidth. We use this technique to measure a triplet $T_{-}$to singlet $S_{0}$ relaxation time of $0.62 \mathrm{~ms}$ in precision donor quantum dots in silicon. We also show that the use of $\mathrm{rf}$ readout does not impact the spin lifetimes ( $S_{0}$ to $T_{-}$decay remained approximately $2 \mathrm{~ms}$ at zero detuning). This establishes single-gate sensing as a viable readout method for spin qubits.
\end{abstract}

DOI: 10.1103/PhysRevX.8.041032

Subject Areas: Mesoscopics,

Quantum Information

\section{INTRODUCTION}

Semiconductor quantum dots show great potential for scalable quantum information processors [1-4]. Singlettriplet qubits, formed by taking the subspace of the twoelectron spin states singlet $S_{0}=(|\uparrow \downarrow\rangle-|\downarrow \uparrow\rangle) / \sqrt{2}$ and triplet $T_{0}=(|\uparrow \downarrow\rangle+|\downarrow \uparrow\rangle) / \sqrt{2}$ under a energy gradient (such as a magnetic-field gradient across two quantum dots), have enabled all-electrical control of qubit rotations while demonstrating immunity to common-mode magnetic field noise $[5,6]$. One of the challenges in scaling up to many qubits is the space needed for the spin sensors required to read out and initialize the individual qubits. An optimal solution has been suggested to use the mandatory gates assigned for qubit control and manipulation as singlegate rf sensors [7-11]. When performing readout, electrons in singlet states oscillate between the dots, giving rise to a measurable quantum capacitance $[8,9,11,12]$. Triplet states cannot oscillate electrons due to Pauli blockade, giving rise to a signal contrast between singlets and any of the triplets. The singlet-triplet subspace spanned by singlet $S_{0}$ and triplet $T_{-}=|\downarrow \downarrow\rangle$ can also be used to read out the spin state of single electron spins. Here, by loading a spin-down electron onto the dot with the lower spin-down ground-state energy, rf readout can be used to measure the spin state of the target electron on the other dot [7]. If the target electron

Published by the American Physical Society under the terms of the Creative Commons Attribution 4.0 International license. Further distribution of this work must maintain attribution to the author(s) and the published article's title, journal citation, and DOI. is spin-down, Pauli blockade prevents it from tunneling across the dots and yields no rf response, while a spin-up electron will form a singlet state with the other electron, giving a nonzero $\mathrm{rf}$ response. The single-gate $\mathrm{rf}$ readout can also be employed to measure a single electron spin on a double quantum dot under a magnetic-field gradient [13]. To date, however, the sensitivity of such single-gate sensors has not been high enough to achieve single-shot readout. Single-shot qubit readout is desirable for running state-ofthe-art error correction codes, in which sequential qubit measurements are advantageous in establishing error syndromes $[14,15]$.

Phosphorus donor quantum dots have recently demonstrated progress towards a scalable quantum computer with independent readout of double quantum dot systems using three-lead single electron transistor (SET) sensors [16-18]. Donor systems are also ideal for hosting singlet-triplet qubits, as they exhibit large $(\sim 8 \mathrm{meV})$ singlet-triplet splittings and long relaxation times $[8,19]$. We previously measured a long $S_{0}$ to $T_{-}$relaxation time of $2 \mathrm{~ms}$ (which ultimately sets the overall measurement time limit) when using a single-gate rf sensor [8]. However, the sensitivity of the resonator circuit was limited by the low quality factor of its Coilcraft 1206CS-821XJE chip inductor $(\mathrm{Q}=175)$. The quality factor of the inductor is important because the resonant circuit matches the impedance of the electron tunneling $\left(\sim 10^{8} \Omega\right)$ with the laboratory transmission line $(50 \Omega)$. This implies that we need a quality factor on the order of $10^{3}$ (as $\mathrm{Q} \approx \sqrt{10^{8} / 50}$ when matched). Recent experiments have shown increased sensitivity by using superconducting inductors that have effective quality factors of up to $800[20,21]$. 


\section{METHOD}

In this paper, we integrate a superconducting inductor into a single-gate donor-based quantum-dot architecture in silicon for single-shot readout. The device shown in Fig. 1(a) (previously measured in Ref. [8]) was fabricated in silicon with the leads and dots defined by atomically placed phosphorus donors using hydrogen-resist scanning tunneling microscope (STM) lithography [22]. Two pairs of quantum dots (D1L, D1U) and (D2L, D2U), with each dot consisting of approximately 3-4 P-donors, are each manipulated by two leads: a reservoir to load electrons and a gate to tune the singlet-triplet state. Single-shot readout was performed on a singlet-triplet state hosted across the dots D2L and D2U, using the resonator connected to reservoir R2. A tunnel junction charge sensor TJ was patterned at the side and connected to a chip inductor resonator to help locate a singlet-triplet charge transition [23]. The resonators were connected to a frequency-multiplexed rf output line $[8,20,24]$.

We have incorporated a 100-nm-thick NbTiN superconducting spiral inductor on a $\mathrm{Si}$ substrate to the singlegate sensor R2 to increase the quality factor for the maximal readout signal [as shown in Fig. 1(b)]. This inductor is a 14-turn spiral, $78 \mathrm{~mm}$ in total length, with a $10-\mu \mathrm{m}$ trace width and a $30-\mu \mathrm{m}$ gap between turns.
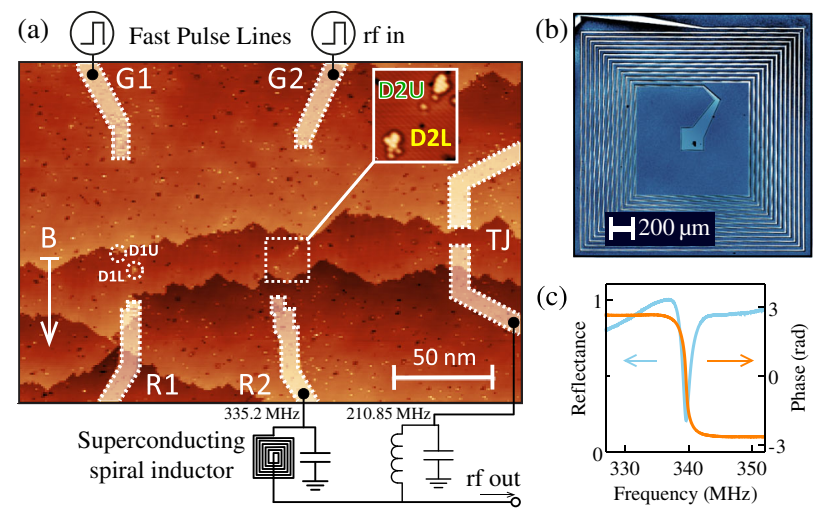

FIG. 1. Single-gate sensor readout with an integrated superconducting resonator circuit. (a) The STM image shows the silicon surface lithography where the lighter regions have been desorbed from the lithographic hydrogen mask. These areas are dosed with phosphorus to form the dots (D1U, D1L, D2U, and D2L) and metallic electrodes [25]. A standard chip inductor connects to the tunnel junction charge sensor TJ. Reservoir R2 is used to load dots D2L and D2U with electrons, while gates G1 and G2 are used to manipulate the singlet-triplet detuning of the dot pairs. The capacitors indicate the parasitic capacitance of the inductors. B indicates the in-plane magnetic field during millikelvin measurements. (b) The superconducting inductor is added to the frequency-multiplexed line, connected to R2, and it measures the singlet-triplet state across D2U and D2L. (c) The reflected (sending and receiving the rf tone via the multiplexed line) frequency response of the superconducting inductor when connected to R2 at zero magnetic field.
Figure 1(c) shows the frequency response of the inductor when connected to R2. The internal and external quality factors of this inductor when wire-bonded to the device (75 mK) were approximately 800 and 400, respectively. The resonator's frequency was $339.6 \mathrm{MHz}$ at zero magnetic field and $335.2 \mathrm{MHz}$ at $2.75 \mathrm{~T}$. The inductor was found to retain its quality factor in parallel magnetic fields of about 3.3 $\mathrm{T}$ due to the large critical field of NbTiN. These large fields are necessary both for operating singlet-triplet qubits (to break the triplet degeneracy) and in performing $\mathrm{rf}$ readout as discussed below.

\section{RESULTS}

In Fig. 2(a), we plot the differential rf amplitude response from the tunnel junction charge sensor TJ as we sweep gates G1 and G2 at the $(3,3)$ to $(2,4)$ interdot charge crossing (the numbers indicate the number of electrons in dots D2L and $\mathrm{D} 2 \mathrm{U}$, respectively). This charge configuration is equivalent spin-wise to a $(1,1)-(0,2)$ singlet-triplet crossing, where we observe a clear interdot transition due to the tunneling of a single electron. We measure the tunnel coupling at the $(3,3)$ to $(2,4)$ transition as $39 \pm 6 \mathrm{GHz}$ by plotting the dependence of the interdot transition at different applied magnetic fields [8]. Since the tunnel coupling is much larger than the driving frequency of the resonator $(335.2 \mathrm{MHz})$, this interdot transition forms a good candidate for single-gate readout, as the rf tone will drive electron oscillations adiabatically between the two dots. We send the rf tone with amplitude $\Delta_{\mathrm{rf}}$ [grey dotted lines in Fig. 2(b)] through $\mathrm{G} 2$, while the superconducting resonator on $\mathrm{R} 2$ captures the resulting $\mathrm{rf}$ response. Measuring the rf response in transmission like this is not fundamentally different from a reflection measurement, nor does it require additional leads. The response was fed into a lock-in amplifier, which modulated the amplitude of the input rf tone at $21.361 \mathrm{kHz}$, to filter the detection of noise originating from the roomtemperature apparatus. The overall measurement bandwidth was approximately $3.3 \mathrm{kHz}$.

When performing single-gate rf readout, triplet states cannot oscillate electrons due to Pauli blockade, while singlet states adiabatically move one electron between the dots [the $S_{0}(1,1)$ and $S_{0}(0,2)$ states], as shown by the red branch in Fig. 2(b). In Fig. 2(c), we show the rf response (measured voltage change in the IQ plane with respect to the background level [24]) as a function of detuning and input rf amplitude $\Delta_{\mathrm{rf}}$ at zero magnetic field (where the singlet is the ground state). We see that the rf response is maximal at zero detuning, as the rf tone moves the electron the greatest distance, pushing it equally into both dots, respectively. We optimized the if amplitude by taking the lowest amplitude that gave the maximum possible signal (at approximately $170 \mu \mathrm{eV}$ ). Increasing the amplitude further only broadens the $\mathrm{rf}$ response in detuning without increasing its magnitude [24]. We set the magnetic field to $2.75 \mathrm{~T}$, as this moves the $S_{0}-T_{-}$anticrossing (the overlap of the red 

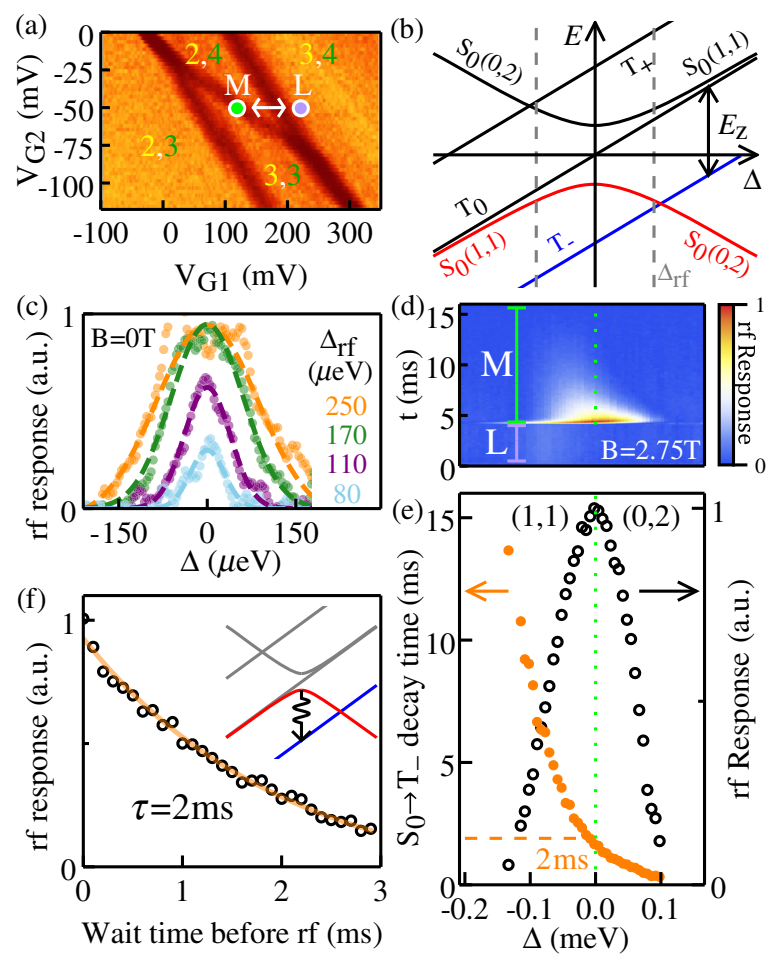

FIG. 2. Optimizing the singlet-triplet readout position. Using the sensor on R2, singlet states were time resolved to find the optimal point in detuning. (a) Charge stability diagram around the $(2,4)-(3,3)$ transition taken by observing the differential rf amplitude response of the sensor TJ while sweeping gates G1 and G2 across a singlet-triplet transition on D2L and D2U. (b) The singlet-triplet energy diagram highlighting the $T_{-}$ground state (blue) and the $S_{0}(1,1)$ to $S_{0}(0,2)$ crossing (red), where the electron oscillates when performing singlet readout. Note that $E_{\mathrm{z}}$ is the Zeeman energy of the triplet $T_{ \pm}$states due to the applied $2.75 \mathrm{~T}$ magnetic field, while $\Delta_{\mathrm{rf}}$ denotes the amplitude of the rf tone. (c) Optimizing the $\mathrm{rf}$ amplitude $\Delta_{\mathrm{rf}}$ at $0 \mathrm{~T}$ to find the minimum amplitude that gives the signal maximum. The dotted lines fit the data with a Gaussian for visual clarity. (d) rf response at $2.75 \mathrm{~T}$ averaged over 10000 shots at different points in detuning. Each trace was taken when waiting at $\mathrm{L}$ for $4.1 \mathrm{~ms}$. The nonzero $\mathrm{rf}$ response signifies the presence of oscillating electrons (singlet states) that eventually decay into triplet $T_{-}$states. (e) When fitting an exponential to each trace, it is clear that, at zero detuning, the rf response is maximal. (f) In all the experiments (a)-(d), the rf tone was constantly present. In this plot, we show the resulting rf response (fitted from the exponential $S_{0}-T_{-}$decays as in (d) at zero detuning when turning on the rf pulse after a time waiting $0-3 \mathrm{~ms}$ at $\mathrm{M}$. The resulting decay (shown in the inset) has the same time constant of $2 \mathrm{~ms}$, suggesting that the decay is not an effect of applying the rf tone.

and blue lines) far enough away from the zero-detuning point such that the rf tone does not drive the detuning to this anticrossing.

If we consider Fig. 2(a), we can load a singlet $S_{0}(0,2)$ state by pulsing from the $(3,4)$ state at point $\mathrm{L}$ to the $(2,4)$ state at point M. It is important that we wait about $4 \mathrm{~ms}$ at point $\mathrm{L}$ before moving to the measurement point $\mathrm{M}$ so that the spin relaxes to the singlet ground state as discussed later. The energy diagram describing the two electrons across the two quantum dots [Fig. 2(b)] shows that, at the zero detuning readout point $\mathrm{M}$, the triplet $T_{-}$(blue line) is the ground state. Thus, the singlet $S_{0}$ will eventually decay into the triplet $T_{-}$state [inset in Fig. 2(f)] during measurement, and this sets an upper bound to the overall measurement time.

To find the optimal singlet-triplet readout point at $2.75 \mathrm{~T}$, we measure the rf response at different points in detuning $\Delta$, as shown in Fig. 2(d), taking an average of 10000 individual time traces at each point. On moving point $\mathrm{M}$ from negative detuning to zero detuning, a nonzero response is observed, indicating the presence of a singlet state. The response decays with time due to the singlet relaxing to the $T_{-}$ground state. This signal decays faster at positive detuning, as the rf tone oscillates past $S_{0}-T_{-}$anticrossing [26]. We fit these decay events at different points in detuning to an exponential distribution, giving the amplitudes and time constants of the decay events in Fig. 2(e) (in black and orange, respectively). Although the $S_{0}-T_{-}$lifetime is longer in negative detuning, the optimal readout point is still at zero detuning, where the signal strength is maximal.

An undesirable side effect of the single-gate sensor would be a reduction in the $S_{0}-T_{-}$lifetime when applying the rf tone (e.g., due to spin-orbit coupling) since we do not want the detector to affect the population dynamics of the singlet-triplet state during measurement. To measure the bare $S_{0}-T_{-}$lifetime when no rf tone is applied, we start with the $\mathrm{rf}$ tone turned off, wait at point $\mathrm{L}$ for $4.1 \mathrm{~ms}$ (to load a singlet as before), and then move to zero detuning. We only switch on the rf tone to measure the singlet population after waiting different time periods at point $\mathrm{M}$, as shown in Fig. 2(f). When fitting to the resulting exponential decay of the singlet population, we find that the decay time remains the same $(2 \mathrm{~ms})$ as that when the rf tone remains switched on during the whole experiment [Fig. 2(e)]. Thus, we conclude that the rf excitation does not play a major role in the $S_{0}-T_{-}$decay, as the singlet lifetime remains unaffected by the rf measurement tone.

We perform single-shot measurements of singlet states (equivalent to detecting a spin-up electron [7]) by waiting at point $\mathrm{L}$ for $4.1 \mathrm{~ms}$ and then pulsing to point $\mathrm{M}$ at zero detuning for maximum rf response. Here, we leave the rf tone switched on and measure the resulting response over time in Fig. 3(a). Five such single-shot time traces are shown in red, demonstrating that we can detect a singlet state. Here, when moving to point $\mathrm{M}$, the signal (dotted lines) clearly departs from the background level (dashed lines). After stochastic relaxation $\left(S_{0} \rightarrow T_{-}\right)$, the signal returns back to the background level, highlighting real-time single-shot spin detection. We show, for comparison, three traces (in blue) measured without pulsing to point $\mathrm{L}$ (that is, always reading a triplet $T_{-}$). In these traces, the signal remains at the background level throughout the measurement. To quantify 
(a)

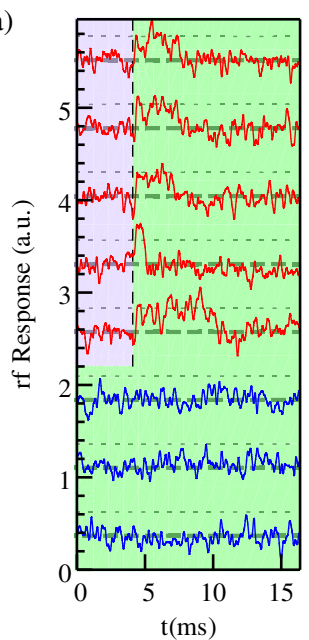

(b)

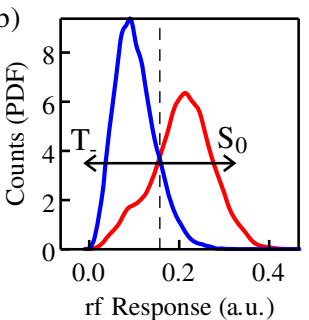

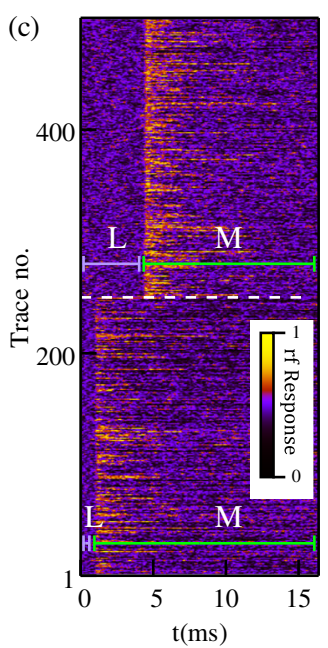

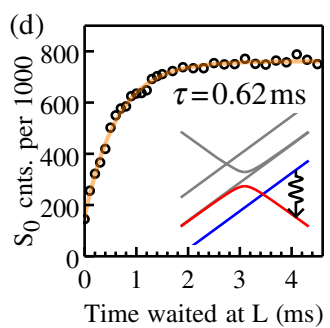

FIG. 3. Single-shot single-spin readout. Using the superconducting spiral inductor on our single-gate sensor R2, single spin states could be resolved with a single shot. (a) Single-shot traces (offset) measuring a single spin in the singlet state when waiting at $\mathrm{L}$ for $4.1 \mathrm{~ms}$ (shown in red) compared to those in triplet states where we did not pulse to L (shown in blue). (b) A histogram [a probability density function (PDF) from 10000 traces] of the maximum value of the rf response when waiting at point $\mathrm{L}$ for $0 \mathrm{~s}$ and $4.1 \mathrm{~ms}$ shown in blue and red, respectively. The dashed line shows the selected threshold that maximizes the readout fidelity at $82.9 \%$. (c) 500 individual time traces of the rf response. The first 250 were taken after waiting at L for $0.7 \mathrm{~ms}$ to partially load singlet states, and the second 250 traces were taken after waiting at $\mathrm{L}$ for $4.1 \mathrm{~ms}$ to fully load singlet states. The high rf response signifies the presence of singlet states that stochastically decay into triplet $T_{-}$states. The shorter wait time highlights the lower singlet population, as insufficient time was given for the $T_{-}$state to decay into the $S_{0}$ state before measurement. (d) To observe this dependence, using the optimal readout threshold, 1000 singleshot traces were taken to measure the singlet population on varying the time spent at point $\mathrm{L}$. This probes the $T_{-}$to $S_{0}$ relaxation time at $\Delta \sim 1 \mathrm{meV}$ of $0.62 \mathrm{~ms}$.

the fidelity of the single-shot spin readout, we must discriminate between a fully null signal (triplet) and one with a nonzero signal (singlet). The singlet signal, on average, follows an exponential decay function [as shown in Fig. 2(e)]; thus, the contrast is concentrated at the beginning of the measurement. Thus, we apply an exponential window over the portion of the signal where the measurement begins (after we have moved to zero detuning at point $\mathrm{M}$ ) and then compile a histogram of the maximum value of each trace [27]. The histogram shown in Fig. 3(b)

was created from 10000 traces taken after waiting at point $\mathrm{L}$ for $4.1 \mathrm{~ms}$ (initializing singlet) and without pulsing to $\mathrm{L}$ (initializing triplet $T_{-}$) to measure the distribution for singlets and triplets, respectively. We take a threshold [shown by the dotted line in Fig. 3(b)] to optimally partition the distributions such that values above are assigned as singlet states and values below are triplet states. This yields an average single-shot readout fidelity of $82.9 \%$ (where the singlet and triplet readout fidelities are $78.2 \%$ and $87.6 \%$, respectively).

With single-shot readout, we measure the $T_{-}$to $S_{0}$ decay at $\Delta=1 \mathrm{meV}$ by varying the time the pulse spends at point L. After every measurement at point $\mathrm{M}$, the electrons decay into the triplet $T_{-}$state. On initially pulsing to point $\mathrm{L}$, the electrons remain in $T_{-}$under the $(3,3)$ charge state. Since the electron tunnel rate from $\mathrm{R} 2$ to $\mathrm{D} 2 \mathrm{U}$ is slow, the system cannot immediately enter the $(3,4)$ charge ground state. The triplet $T_{-}$state must decay into the singlet state [the $(2,4)$ charge state] before an electron can move from R2 to D2L to leave system in the $(3,4)$ state $[8,28]$. Figure $3(\mathrm{c})$ shows 250 traces taken when waiting $0.7 \mathrm{~ms}$ at point $\mathrm{L}$ and 250 traces when waiting $4.1 \mathrm{~ms}$ at point $\mathrm{L}$. The lengths of each nonzero signal are exponentially distributed with a time constant of $2 \mathrm{~ms}$, and they represent singlet states decaying into triplet $T_{-}$states. When waiting a lower time at $\mathrm{L}$, there is clearly a smaller proportion of singlet states. Figure 3(d) shows the singlet counts over 1000 traces taken at different wait times at point $\mathrm{L}$. When viewing the singlet counts as a function of the wait time at point $\mathrm{L}$ and fitting to the resulting exponential rise in the singlet counts, the decay time was measured to be $0.62 \mathrm{~ms}$. Thus, waiting $4.1 \mathrm{~ms}$ at point $\mathrm{L}$ ensures high-fidelity initialization of singlet states.

\section{CONCLUSION}

In summary, we demonstrated single-shot electron spin readout in the singlet-triplet basis, in silicon, using a singlegate $\mathrm{rf}$ sensor. The reduction in gate density using a singlegate sensor simplifies architectures for large arrays of solidstate qubits $[7,8]$. We demonstrated that the $S_{0}$ to $T_{-}$ relaxation time, which limits the qubit measurement time, is $2 \mathrm{~ms}$ and unaffected by the presence of the rf tone. The single-gate rf sensor gives an average measurement fidelity of $82.3 \%$ at $3.3 \mathrm{kHz}$ bandwidth. The fidelity can be improved in future experiments by increasing the internal quality factor, which may be limited by dielectric losses, radiative losses, and/or defects in the 100-nm NbTiN films. These can be improved by using an optimized NbTiN film and substrate [29] and by reducing the overall size of the inductor [30]. The external quality factor can be optimized by redesigning the superconducting resonator geometry to achieve the ideal coupling to the transmission line [31]. One of the challenges in scaling to many qubits using this approach is the space real estate needed for these matching circuits. While we have presented results with a superconducting spiral inductor of a fairly large footprint $\left(4 \mathrm{~mm}^{2}\right)$, this is mainly limited by the 
need for a central bond pad to keep the fabrication simple. It has recently been proposed that these inductors can be further scaled with thinner tracks and tighter spacings to achieve a density of thousands of resonators per square centimeter [20]. Thus, the use of single-gate rf readout now presents a viable option in a scalable quantum computer as also seen recently by other groups $[32,33]$.

\section{ACKNOWLEDGMENTS}

This research is supported by the Australian Research Council Centre of Excellence for Quantum Computation and Communication Technology (Project No. CE110001027) and the U.S. Army Research Office under Contract No. W911NF-17-1-0202. The device was fabricated in part at the New South Wales node of the Australian National Fabrication Facility. We also thank Lucas Orona for useful discussions.

[1] D. Loss and D. P. DiVincenzo, Quantum Computation with Quantum Dots, Phys. Rev. A 57, 120 (1998).

[2] J. Levy, Universal Quantum Computation with Spin-1/2 Pairs and Heisenberg Exchange, Phys. Rev. Lett. 89, 147902 (2002).

[3] R. Hanson, L. P. Kouwenhoven, J. R. Petta, S. Tarucha, and L. M. K. Vandersypen, Spins in Few-Electron Quantum Dots, Rev. Mod. Phys. 79, 1217 (2007).

[4] F. A. Zwanenburg, A. S. Dzurak, A. Morello, M. Y. Simmons, L. C. L. Hollenberg, G. Klimeck, S. Rogge, S. N. Coppersmith, and M. A. Eriksson, Silicon Quantum Electronics, Rev. Mod. Phys. 85, 961 (2013).

[5] J. R. Petta, Coherent Manipulation of Coupled Electron Spins in Semiconductor Quantum Dots, Science 309, 2180 (2005).

[6] M. D. Shulman, O. E. Dial, S. P. Harvey, H. Bluhm, V. Umansky, and A. Yacoby, Demonstration of Entanglement of Electrostatically Coupled Singlet-Triplet Qubits, Science 336, 202 (2012).

[7] M. Veldhorst, H. G. J. Eenink, C. H. Yang, and A. S. Dzurak, Silicon CMOS Architecture for a Spin-based Quantum Computer, Nat. Commun. 8, 1766 (2017).

[8] P. Pakkiam, M. G. House, M. Koch, and M. Y. Simmons, Characterization of a Scalable Donor-based Singlet-Triplet Qubit Architecture in Silicon, Nano Lett. 18, 4081 (2018).

[9] K. D. Petersson, C. G. Smith, D. Anderson, P. Atkinson, G. A. C. Jones, and D. A. Ritchie, Charge and Spin State Readout of a Double Quantum Dot Coupled to a Resonator, Nano Lett. 10, 2789 (2010).

[10] J. I. Colless, A. C. Mahoney, J. M. Hornibrook, A. C. Doherty, H. Lu, A. C. Gossard, and D. J. Reilly, Dispersive Readout of a Few-Electron Double Quantum Dot with Fast rf Gate Sensors, Phys. Rev. Lett. 110, 046805 (2013).

[11] M. G. House, T. Kobayashi, B. Weber, S. J. Hile, T. F. Watson, J. van der Heijden, S. Rogge, and M. Y. Simmons, Radio Frequency Measurements of Tunnel Couplings and Singlet-Triplet Spin States in Si:P Quantum Dots, Nat. Commun. 6, 8848 (2015).
[12] M. D. Schroer, M. Jung, K. D. Petersson, and J. R. Petta, Radio Frequency Charge Parity Meter, Phys. Rev. Lett. 109, 166804 (2012).

[13] X. Mi, M. Benito, S. Putz, D. M. Zajac, J. M. Taylor, G. Burkard, and J. R. Petta, A Coherent Spin-Photon Interface in Silicon, Nature (London) 555, 599 (2018).

[14] A. G. Fowler, M. Mariantoni, J. M. Martinis, and A. N. Cleland, Surface Codes: Towards Practical Large-Scale Quantum Computation, Phys. Rev. A 86, 032324 (2012).

[15] E. T. Campbell, B. M. Terhal, and C. Vuillot, Roads Towards Fault-Tolerant Universal Quantum Computation, Nature 549, 172 (2017).

[16] T. F. Watson, B. Weber, Y.-L. Hsueh, L. C. L. Hollenberg, R. Rahman, and M. Y. Simmons, Atomically Engineered Electron Spin Lifetimes of $30 \mathrm{~s}$ in Silicon, Sci. Adv. 3, e1602811 (2017).

[17] M. A. Broome, S. K. Gorman, M. G. House, S. J. Hile, J. G. Keizer, D. Keith, C. D. Hill, T. F. Watson, W. J. Baker, L. C. L. Hollenberg, and M. Y. Simmons, Two-Electron Spin Correlations in Precision Placed Donors in Silicon, Nat. Commun. 9, 980 (2018).

[18] S. J. Hile, L. Fricke, M. G. House, E. Peretz, C. Y.Chen, Y. Wang, M. Broome, S. K. Gorman, J. G. Keizer, R. Rahman, and M. Y. Simmons, Addressable Electron Spin Resonance Using Donors and Donor Molecules in Silicon, Sci. Adv. 4, eaaq1459 (2018).

[19] B. Weber, Y. H. Matthias Tan, S. Mahapatra, T. F. Watson, H. Ryu, R. Rahman, L. C. L. Hollenberg, G. Klimeck, and M. Y. Simmons, Spin Blockade and Exchange in CoulombConfined Silicon Double Quantum Dots, Nat. Nanotechnol. 9, 430 (2014).

[20] J. M. Hornibrook, J. I. Colless, A. C. Mahoney, X. G. Croot, S. Blanvillain, H. Lu, A. C. Gossard, and D. J. Reilly, Frequency Multiplexing for Readout of Spin Qubits, Appl. Phys. Lett. 104, 103108 (2014).

[21] I. Ahmed, J. A. Haigh, S. Schaal, S. Barraud, Y. Zhu, C.-m. Lee, M. Amado, J. W. A. Robinson, A. Rossi, J. J. L. Morton, and M.F. Gonzalez-Zalba, Radio-Frequency Capacitive Gate-Based Sensing, Phys. Rev. Applied 10, 014018 (2018).

[22] M. Fuechsle, J. A. Miwa, S. Mahapatra, H. Ryu, S. Lee, O. Warschkow, L. C. L. Hollenberg, G. Klimeck, and M. Y. Simmons, A Single-Atom Transistor, Nat. Nanotechnol. 7, 242 (2012).

[23] M. G. House, E. Peretz, J. G. Keizer, S. J. Hile, and M. Y. Simmons, Single-Charge Detection by an Atomic Precision Tunnel Junction, Appl. Phys. Lett. 104, 113111 (2014).

[24] M. G. House, I. Bartlett, P. Pakkiam, M. Koch, E. Peretz, J. van der Heijden, T. Kobayashi, S. Rogge, and M. Y. Simmons, High-Sensitivity Charge Detection with a Single-Lead Quantum Dot for Scalable Quantum Computation, Phys. Rev. Applied 6, 044016 (2016).

[25] B. Weber, S. Mahapatra, H. Ryu, S. Lee, A. Fuhrer, T. C. G. Reusch, D. L. Thompson, W. C. T. Lee, G. Klimeck, L. C. L. Hollenberg, and M. Y. Simmons, Ohm's Law Survives to the Atomic Scale, Science 335, 64 (2012).

[26] S. K. Gorman, M. A. Broome, M. G. House, S. J. Hile, J. G. Keizer, D. Keith, T. F. Watson, W. J. Baker, and M. Y. Simmons, Singlet-Triplet Minus Mixing and Relaxation Lifetimes in a Double Donor Dot, Appl. Phys. Lett. 112, 243105 (2018). 
[27] B. D'Anjou and W. A. Coish, Optimal Post-Processing for a Generic Single-Shot Qubit Readout, Phys. Rev. A 89, 012313 (2014).

[28] C. H. Yang, A. Rossi, N. S. Lai, R. Leon, W. H. Lim, and A.S. Dzurak, Charge State Hysteresis in Semiconductor Quantum Dots, Appl. Phys. Lett. 105, 183505 (2014).

[29] R. Barends, N. Vercruyssen, A. Endo, P. J. de Visser, T. Zijlstra, T. M. Klapwijk, P. Diener, S. J. C. Yates, and J. J. A. Baselmans, Minimal Resonator Loss for Circuit Quantum Electrodynamics, Appl. Phys. Lett. 97, 023508 (2010).

[30] J. Zmuidzinas, Superconducting Microresonators: Physics and Applications, Annu. Rev. Condens. Matter Phys. 3, 169 (2012).
[31] O. Noroozian, Superconducting Microwave Resonator Arrays for Submillimeter/Far-Infrared Imaging, Ph.D. thesis, California Institute of Technology, 2012.

[32] A. West, B. Hensen, A. Jouan, T. Tanttu, C. H. Yang, A. Rossi, M. F. Gonzalez-Zalba, F. E. Hudson, A. Morello, D. J. Reilly, and A. S. Dzurak, Gate-based Single-Shot Readout of Spins in Silicon, arXiv:1809.01864.

[33] M. Urdampilleta, D. J. Niegemann, E. Chanrion, B. Jadot, C. Spence, P.-A. Mortemousque, C. Buerle, L. Hutin, B. Bertrand, S. Barraud, R. Maurand, M. Sanquer, X. Jehl, S. De Franceschi, M. Vinet, and T. Meunier, Gate-based High Fidelity Spin Read-out in a CMOS Device, arXiv: 1809.04584 . 